\title{
Preface
}

\section{Large amplitude internal waves in the coastal ocean}

\author{
R. Grimshaw ${ }^{1}$, K. Helfrich ${ }^{2}$, and A. Scotti ${ }^{3}$ \\ ${ }^{1}$ Department of Mathematical Sciences, Loughborough University, Leicestershire, UK \\ ${ }^{2}$ Woods Hole Oceanographic Institution, Woods Hole, USA \\ ${ }^{3}$ Department of Marine Sciences, University of North Carolina, USA
}

The flow in the coastal ocean, and especially on the continental shelf and slope is often characterized by the presence of very large-amplitude internal waves. These are waves which occur in the interior of the ocean, and propagate horizontally with a concentration of their energy around the oceanic pcynocline. They are usually generated by the interaction of the barotropic tide with the shelf break, topographic sill or with other prominent bottom features. This leads to the formation of an internal tide, which then deforms and evolves into a train of very large-amplitude internal waves, with associated large pycnocline displacements and strong currents. They are highly significant for sediment transport and for the biology on the continental shelf, their associated currents cause strong forces on marine platforms and submersibles, the associated strong distortion of the density field has a severe impact on acoustic signaling and their capacity to break and form microstructure has major consequences for the understanding of interior ocean mixing.

It is now well established that the observed waves can be understood as internal solitary waves, or as internal undular bores, and to a first level of understanding can be modeled by the Korteweg-de Vries (KdV) equation, or by one of various extensions, see the reviews by Grimshaw $(2001,2010)$ and Helfrich and Melville (2006). While these models form the basic paradigm, there remain several outstanding issues which are of current concern. These include estimating the effects of shear-induced instability, a need to go beyond the weakly nonlinear $\mathrm{KdV}$ models and study finite-amplitude waves, the effect of background rotation, transverse effects induced by topography, and the need to go beyond the single

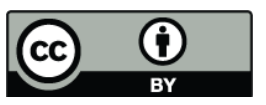

Correspondence to: R. Grimshaw (r.h.j.grimshaw@lboro.ac.uk) mode assumption which characterizes the KdV models. This special issue is intended to provide a sample of recent work which addresses some of these and other related issues. There were eleven papers in the special issue, which we now discuss in the order of their publication.

Helfrich and White (2010) construct steady largeamplitude internal solitary waves in a continuously stratified fluid by solving the Dubreil-Jacotin-Long (DJL) equation numerically. Sufficiently large waves may develop recirculating cores. As previously modeled, these recirculating cores often contained densities outside the ambient range, which are statically unstable, and hence the solutions were probably not physically realizable. To address this issue, in this paper the trapped core of unknown shape is assumed to have constant density and zero vorticity. The results show that small-amplitude solutions without recirculating cores can be continued along a solution branch as the amplitude increases into a regime with a stagnant core. Numerical solutions of the time-dependent equations initiated with these stagnant-core solutions show that the waves are stable up to a critical amplitude above which shear instability occurs.

As mentioned earlier, nonlinear internal waves can contribute a significant fraction of the mixing and dissipation observed over continental shelves. Shroyer et al. (2010) analyze data obtained over the New Jersey shelf during the summer of 2006. Nonlinear internal waves were observed using a combination of moorings and shipboard observations. The latter was used to follow the evolution of individual wave packets as they raced across the shelf. The observations indicate that the waves carry energy of the order of $10^{5} \mathrm{~J} \mathrm{~m}^{-1}$ and dissipate about $50 \mathrm{~W} \mathrm{~m}^{-1}$, although values twice as large were observed during part of the deployment period. The waves were followed from the outer shelf where they form to the inner shelf where they dissipate. They were observed to undergo an initial period of growth, after which

Published by Copernicus Publications on behalf of the European Geosciences Union and the American Geophysical Union. 
decay followed. The measured dissipation correlates with the energy density of the waves, and was attributed to shear instabilities.

Most theoretical models and interpretation of oceanic data are based on the assumption of horizontal propagation in a single horizontal direction. McMillan and Sutherland (2010) describe a laboratory experiment in which an axisymmetric intrusion into a two-layer fluid generates an axisymmetric second mode internal solitary wave propagating radially outward in the $r$-direction. Although a cylindrical $\mathrm{KdV}$ model is available to describe such waves, they find that this model is only asymptotically valid for a very large radial distance, when linear dispersion starts to dominate over nonlinearity. needs to be adjusted to account for the observed radial decay of $r^{-1 / 2}$ of the solitary wave. Consequently they heuristically adjusted the cylindrical KdV model so that the solitary wave solutions decay as observed.

Ramp et al. (2010) present results from a fourteen month deployment of four oceanographic moorings spanning the South China Sea, a region of recent interest due to the presence of very large amplitude internal waves. These observations show that the internal tide is generated within the Luzon Strait and propagates to the WNW. The nonlinear internal waves emerge from the tide as it propagates across the basin. Waves with different characteristics are associated with both the strength and frequency (diurnal and semidiurnal) of the tidal forcing in the Luzon Strait. The nonlinear waves, but not the tides, are absent during the winter months.

Internal tide and wave modeling is often considered in the context of a single vertical mode. Vlasenko et al. (2010) relax this assumption in a numerical modeling study of the internal tide in the South China Sea. The numerical solutions illustrate the role of two adjacent ridges on the multimodal character of the radiated internal tide and subsequent internal waves. Specifically, the shallower western ridge is shown to be the dominant source of the higher-mode waves. The interaction of the internal tides of different modes is responsible for the generation of an unusual waveform in which a high wavenumber, first-mode wavetrain is embedded in an envelop with second-mode structure.

Interaction of nonlinear internal waves with background shear occurs frequently in the coastal ocean. Lamb (2010) uses numerical simulations to investigate the energetics of waves interacting with a sheared background flow. The total energy of the waves is partitioned between available potential energy and kinetic energy. The paper explores the difficulty associated in defining an appropriate averaging procedure to separate background from wave and turbulent flow. For slowly varying linear internal waves, spatial averaging over several wavelengths accomplishes the task. However, for nonlinear, pulse-like waves, such averaging does not make sense, and the author argues that a logical choice is to consider the far upstream field velocity as the background state. Several different combinations of waves and topography are considered. The results show that surface waves (normally disregarded in this type of simulation) may play an important role in the energetics of waves interacting with topography, calling for a more careful analysis.

Among the several mechanisms for the generation of an internal tide, some recent observations in the Bay of Biscay, Mozambique Channel and the Mascarene Ridge have provided evidence that an an internal tidal beam impinging on the pycnocline from below can generate internal solitary waves riding on this pycnocline. Grisouard and Staquet (2010) report numerical simulations of this process for Bay of Biscay summer conditions. Although they find that the mechanism is viable, the internal solitary wave amplitudes found differ from those observed. However, when the background current fields are included in the numerical model, much better agreement is obtained. This is a rather instance in the modeling of internal solitary waves when the effects of a background current have been found to be significant.

Most observations of internal solitary waves are for mode one, which has the fastest phase speed. However, recently there have been a few reports of mode two waves. Although mode two internal solitary waves cannot normally exist as steady travelling waves due to a resonance with mode one short waves, they can be generated as transients by interaction with topography. Yang et al. (2010) report observations of mode two waves on the continental slope of the South China Sea, and apparently for the first time, found two distinct types. The usual mode two wave typically has a convex form with a bulge in the pycnocline, and this kind is that usually found, However, the observations reported here show a few cases of a mode two wave with a convex form, with a localized thinning ("anti-bulge") of the pycnocline. The theoretical explanation, based on the KdV equation, is that for a three-layer fluid structure, a convex mode two wave is found when the middle layer is thin, and a concave mode two wave is found when the middle layer is thick. This latter case is rather unusual on the coastal ocean.

Large-amplitude internal waves are not limited to the ocean. Magalhaes et al. (2011) use satellite imagery to identify a new hotspot in the Red Sea of nonlinear internal waves propagating in the lower troposphere. Historical atmospheric observations are used to demonstrate that the vertical trapping required for horizontal propagation exists. The satellite observations of wave propagation speeds are consistent with a weakly-nonlinear KdV and Benjamin-Ono wave models. The analysis also highlights the care that must be exercised to distinguish oceanic from atmospheric internal waves in ocean surface satellite imagery.

Typically, nonlinear waves require both nonlinearity and dispersion. However, it often occurs that the latter becomes important only after the former has had time to generate steep wavefronts, thus dispersion may be neglected during the early stage of development of an internal tide. Ostrovsky and Helfrich (2011) focus on solutions for nonlinear and 
non-dispersive internal waves in arbitrary stratification. Well known solutions exist for the single-layer (surface waves) and two-layer case, in which the speed of any point along the interface depends only on the amplitude of the local perturbation. The existence of these "simple" waves in arbitrary stratification is investigated by the authors, using a combination of analytical calculations and numerical solutions. They show that it is possible to have such "simple" wave solutions.

The topic of nonlinear internal wave generation and the classic dead-water phenomenon is explored in a laboratory study by Mercier et al. (2011). In the experiments a small boat is towed with a constant force on the surface of a stratified fluid, thus permitting the speed of the boat to vary in response to changes in wave drag from the forced internal waves. Regimes of oscillatory boat motion occur for sub-critical mean boat speeds. In stratifications that permit multiple vertical modes, the boat motion is tightly coupled to only the lowest vertical mode. Wave amplitudes can be large with solitary-like waves present, as has been found previously for steady forcing in the transcritical regime.

\section{References}

Grimshaw, R.: Internal solitary waves. In: Environmental Stratified Flows, edited by: Grimshaw, R., Kluwer, Boston, Chapter 1, 128, 2001.

Grimshaw, R., Pelinovsky, E., Talipova, T., and Kurkina, O.: Internal solitary waves: propagation, deformation and disintegration, Nonlin. Processes Geophys., 17, 633-649, doi:10.5194/npg-17633-2010, 2010.

Grisouard, N. and Staquet, C.: Numerical simulations of the local generation of internal solitary waves in the Bay of Biscay, Nonlin. Processes Geophys., 17, 575-584, doi:10.5194/npg-17575-2010, 2010.
Helfrich, K. R. and Melville, W. K.: Long nonlinear internal waves, Annu. Rev. Fluid Mech., 38, 395-425, 2006.

Helfrich, K. R. and White, B. L.: A model for large-amplitude internal solitary waves with trapped cores, Nonlin. Processes Geophys., 17, 303-318, doi:10.5194/npg-17-303-2010, 2010.

Lamb, K. G.: Energetics of internal solitary waves in a background sheared current, Nonlin. Processes Geophys., 17, 553-568, doi:10.5194/npg-17-553-2010, 2010.

Magalhaes, J. M., Araújo, I. B., da Silva, J. C. B., Grimshaw, R. H. J., Davis, K., and Pineda, J.: Atmospheric gravity waves in the Red Sea: a new hotspot, Nonlin. Processes Geophys., 18, 71-79, doi:10.5194/npg-18-71-2011, 2011.

McMillan, J. M. and Sutherland, B. R.: The lifecycle of axisymmetric internal solitary waves, Nonlin. Processes Geophys., 17, 443-453, doi:10.5194/npg-17-443-2010, 2010.

Mercier, M. J., Vasseur, R., and Dauxois, T.: Resurrecting deadwater phenomenon, Nonlin. Processes Geophys., 18, 193-208, doi:10.5194/npg-18-193-2011, 2011.

Ostrovsky, L. A. and Helfrich, K. R.: Strongly nonlinear, simple internal waves in continuously-stratified, shallow fluids, Nonlin. Processes Geophys., 18, 91-102, doi:10.5194/npg-18-91-2011, 2011.

Ramp, S. R., Yang, Y. J., and Bahr, F. L.: Characterizing the nonlinear internal wave climate in the northeastern South China Sea, Nonlin. Processes Geophys., 17, 481-498, doi:10.5194/npg-17481-2010, 2010.

Shroyer, E. L., Moum, J. N., and Nash, J. D.: Energy transformations and dissipation of nonlinear internal waves over New Jersey's continental shelf, Nonlin. Processes Geophys., 17, 345-360, doi:10.5194/npg-17-345-2010, 2010.

Vlasenko, V., Stashchuk, N., Guo, C., and Chen, X.: Multimodal structure of baroclinic tides in the South China Sea, Nonlin. Processes Geophys., 17, 529-543, doi:10.5194/npg-17-5292010, 2010.

Yang, Y. J., Fang, Y. C., Tang, T. Y., and Ramp, S. R.: Convex and concave types of second baroclinic mode internal solitary waves, Nonlin. Processes Geophys., 17, 605-614, doi:10.5194/npg-17605-2010, 2010. 